\title{
Correction to: Neural correlates of reward magnitude and delay during a probabilistic delay discounting task in alcohol use disorder
}

\author{
Laura E. Dennis ${ }^{1,2,3} \cdot$ Milky Kohno ${ }^{1,2,3} \cdot$ Holly D. McCready ${ }^{1,2,3} \cdot$ Daniel L. Schwartz ${ }^{4}$ Britta Schwartz ${ }^{1,2,3}$. \\ David Lahna ${ }^{4}$ • Bonnie J. Nagel ${ }^{2,3}$ • Suzanne H. Mitchell ${ }^{2,3,5}$ • William F. Hoffman 1,2,3,6
}

Published online: 21 December 2019

(C) Springer-Verlag GmbH Germany, part of Springer Nature 2019

\section{Correction to: Psychopharmacology}

https://doi.org/10.1007/s00213-019-05364-3

After publication of this paper, the authors determined an error in the funding information section CX17008-CDA2 should be CX001790 (MK). Additionally, the following funding source was omitted in the original publication but will be added here: Oregon Health \& Science University Center for Women's Health - Circle of Giving APSYC0287.

Publisher's note Springer Nature remains neutral with regard to jurisdictional claims in published maps and institutional affiliations.

The online version of the original article can be found at https://doi.org/ 10.1007/s00213-019-05364-3

Milky Kohno

kohno@ohsu.edu

1 Mental Health Division P35C, Veterans Affairs Portland Health Care System, 3710 SW US Veterans Hospital Rd, Portland, OR 97239, USA

2 Department of Psychiatry, Oregon Health \& Science University, Portland, OR, USA

3 Department of Behavioral Neuroscience, Oregon Health \& Science University, Portland, OR, USA

4 Neurology and Advanced Imaging Research Center, Oregon Health \& Science University, Portland, OR, USA

5 Oregon Institute for Occupational Health Sciences, Oregon Health \& Science University, Portland, OR, USA

6 Methamphetamine Abuse Research Center (MARC), Oregon Health $\&$ Science University and Veterans Affairs Portland Health Care System, Portland, OR, USA 